\title{
Statistical framework of the inverse problem in the retrieval of vegetation parameters
}

\author{
Bruno CombaL*, Sergey L. OchSHEPKov, Alexander SinYuK, Harumi IsAKA \\ Laboratoire de Météorologie Physique (LaMP), Université Blaise Pascal, 24 avenue des Landais, \\ 63177 Aubière Cedex, France
}

(Received 3 June 1999; accepted 22 November 1999)

\begin{abstract}
The accuracy of the vegetation properties retrieved from radiometric data depends mainly on the information about the target parameters contained within each measurement. We postulate that a measurement provides the more information the more it contributes to decrease the uncertainty in the retrieved parameters. The mathematical definition of the information contents based on the statistical estimation theory, reveals clearly the role played by both the sensitivities of the measurements with respect to the desired parameters and the correlation between these sensitivities.

In this paper, we discuss especially the application of this formal framework to the retrieval of the vegetation properties, and investigate some fundamental problems such as the limitation of the accuracy of the target parameters in their retrieval. We also show how this limitation depends on the biome properties and on the geometrical configuration of the illumination-observation.

Furthermore, it should be emphasised that the present formalism provides a theoretical framework to treat different aspects of the retrieval of medium properties. In particular, it enables to define how to organise sampling of most informative measurements.
\end{abstract}

\section{Remote Sensing / information contents / inverse problem / accuracy / vegetation}

Résumé - Cadre statistique du problème inverse pour la restitution de paramètres de la végétation. La précision des propriétés de la végétation estimées à partir d'un jeu de données radiométriques dépend principalement de l'information relative aux paramètres de la cible contenue dans chacune des mesures. On peut considérer qu'une mesure fournit d'autant plus d'information qu'elle contribue à diminuer l'incertitude relative aux paramètres inversés. La définition mathématique du contenu en information, basée sur la théorie de l'estimation statistique, montre clairement le rôle joué à la fois par la sensibilité des mesures aux paramètres que l'on désire estimer et par les corrélations de ces sensibilités. Dans cet article, nous discutons l'application de ce formalisme à la restitution des propriétés de la végétation et examinons quelques problèmes fondamentaux tels que la limitation de la précision de l'estimation des propriétés de la cible. Nous montrons aussi comment ces limitations dépendent des propriétés du milieu végétal et de la configuration géométrique des angles d'éclairage et d'observation.

Communicated by Andres Kuusk (Toravere, Estonia)

* Correspondence and reprints

combal@opgc.univ-bpclermont.fr 
De plus, il faut souligner que ce formalisme fournit un cadre théorique permettant de traiter différents aspects de la restitution des propriétés d'un milieu. En particulier, il permet de définir l'organisation de l'échantillonnage des mesures contenant le plus d'information.

Télédétection / contenu en information / problème inverse / précision / végétation

\section{Introduction}

New radiometers offer a variety of observational capabilities of the Earth surface. For example, MISR (Multiangle Imaging SpectroRadiometer) can provide radiance measurements for nine view angles and four wavelengths, MODIS (MODerate resolution Imaging Spectro-radiometer) for a single angle of view and seven wavelengths, and POLDER (POLarisation and Directionality of the Earth's Reflectances) measures radiances for 12 to 14 viewing angles, eight wavelengths and polarisation for some wavelengths. Consequently, one of the major issues of the remote sensing is how to use effectively this variety of radiometric data for the retrieval of parameters characterising the observed medium, but also to improve the procedure of the retrieval in accordance with available data.

In the usual retrieval procedure, one considers that the target vegetation parameters are found when the radiation fields that are computed according to an inversion model agree with the measured radiation fields within a prescribed accuracy. From the statistical point of view, the most general way to characterise the retrieved model parameters is to investigate directly the probability density function of retrieved model parameters, i.e. the 'a posteriori information' on the retrieved model parameters [4, 8]. The inverse problem is based on this posterior probability density function and its solution is to find the maximum likelihood of the model parameters with respect to observation data. However, the inverse problem is frequently solved, in practice, according to the least mean square method [7].

The accuracy of the retrieved parameters depends on the sampling conditions such as sun position, viewing angles and spectral bands [8]. It also depends on the sensitivity of the measurements with respect to the target vegetation properties. An evident fact is that the retrieval will be more accurate as the measurements provide more information about the target parameters. Hence, any appropriate retrieval algorithm should be written in such a way that more weight is given to the most informative measurements. This is usually done by using the weighted least mean square (e.g. see [12] for a general review), in which each weight is defined according to the accuracy of each available measurement.

The sensitivity of the measurements to the vegetation properties changes with the viewing direction and wavelength. In other words, a particular direction or wavelength should be preferred to others for the retrieval of a given surface property. To do so, we need to define and quantify, in some way, the information contents of measurements and to assess an optimal configuration of the satellite for the retrieval of the desired parameters. Such a definition should help also to achieve two objectives: to estimate the information contents available within the data provided by different sensors, and to optimise the satellite observational capability with respect to the parameters. This requires the above defined 'posterior' probability density function to solve the inverse problem by finding the maximum of this probability. Knyazikhin et al. (1998) [4] proposed a method to find the most probable solution of the inverse problem based on the statistical characterisation of the multiple solutions. In this method, the multiple solutions result from the use of a look-up table in the retrieval procedure, and not from the intrinsic properties of the inverse problem itself. The main difference between these two approaches resides in the fact that we define the information contents of the observations within a formal mathematical framework, while Knyazikhin et al. [4] based their 
approach on a statistical characterisation of multiple solutions.

In earlier work, several investigations have been made to estimate the sensitivity of some radiative transfer models to the biome characteristics, e.g. the sensitivity to the LAI [2]. However, the nonlinear dependency of radiation fields to the medium parameters implies some correlations between the sensitivities of various parameters; this means that the accuracy of retrieved parameters cannot be determined only by a sensitivity analysis.

In the present study, we developed a general mathematical framework based on statistical estimation theory to quantify the information contents of radiometric measurements with respect to a given inverse problem. The retrieval of vegetation parameters from multi-angular radiance data in visible and near infrared was analysed within this theoretical framework, by using a simple 1D physical model of the bi-directional reflectance factor (BRF). We evaluated how the correlations between the sensitivities affects the accuracy of the retrieved vegetation parameters and discussed the optimisation of radiometric data sampling.

The formalism presented in this paper should not be confused with the problem of searching the solutions of the inverse problem [4]. We only assess the accuracy of the vegetation parameters for inaccurate measurements and a simplified model with respect to the sampling condition, without considering the problem of computing the solution of the inverse problem.

\section{Materials and methods}

A vegetation model to be used for the parameter retrieval should fulfil a certain number of requirements. It should include in principle, as many physical parameters as needed to represent physically pertinent characteristics of real vegetation canopy. A method of retrieval should also enable to retrieve a set of physical parameters as 'independent' as possible from the select particular set of radiometric data. However, this does not mean that we can include as many parameters as possible.
Let $\mathbf{f}(\mathbf{a})$ be a non-linear operator that associates a vegetation parameter vector a to the predicted data space (simulated BRF). A vector $\mathbf{f}^{*}$ is the vector whose components are the radiances observed at different viewing directions. The components of the vectors $\mathbf{f}(\mathbf{a}), \mathbf{f}^{*}$ and $\mathbf{a}$ are logarithms of simulated and observed radiance, and vegetation parameters. The inverse problem can be defined as finding a solution a such as:

$$
\mathbf{f}^{*}=\mathrm{f}(\mathrm{a})+\xi
$$

where $\xi$ is the error in the simulated values. Generally, the predicted values cannot be identical to the true 'observed' values because of two sources of error: experimental uncertainties and modelling errors. When both the experimental and modelling errors are represented by lognormal distributions, the 'posterior' probability density function in the model space $\sigma_{\mathrm{M}}(\mathbf{a})$ is given by a lognormal distribution function [10]:

$$
\begin{aligned}
\sigma_{\mathrm{M}}(\mathbf{a}) \propto \exp \left[-\frac{1}{2}\left(\left(\mathbf{f}(\mathbf{a})-\mathbf{f}^{*}\right)^{\mathrm{T}} \mathbf{W}^{-1}\left(\mathbf{f}(\mathbf{a})-\mathbf{f}^{*}\right) .\right.\right. \\
\left.+(\mathbf{a}-\mathbf{a} *)^{\mathrm{T}} \mathbf{C}^{-1}(\mathbf{a}-\mathbf{a} *)\right)
\end{aligned}
$$

where $\mathbf{W}$ is the matrix of measurements and modelling errors, and $\mathbf{C}$ is the covariance matrix of the 'a priori' information. A log normal distribution is chosen because the vegetation parameters are strictly positive. The use of usual normal distribution is not recommended because it allows negative values for these strictly positives parameters.

Let us assume that the forward equation (Eq. (1)) can be linearized around the maximum likelihood point $\mathbf{a}_{\infty}$. Then, we can formulate the probability density distribution (Eq. (2)) in the neighbourhood of $\mathbf{a}_{\infty}$, and the covariance matrix of this distribution can be given by $[1,10]$ :

$$
\Delta=\left(\mathbf{U}_{\infty}^{\mathrm{T}} \mathbf{W}^{-1} \mathbf{U}_{\infty}+\mathrm{C}^{-1}\right)^{-1}
$$


where $\mathbf{U}$ is the matrix whose elements are the sensitivity of the measurements to the vegetation parameters [1]:

$$
\{\mathbf{U}\}_{j i}=\frac{\partial f_{j}}{\partial a_{i}} .
$$

The posterior covariance operator $\Delta$ defines completely the posterior probability density distribution of the error in the retrieved vegetation parameters. It also enables to analyse how the information about the vegetation properties is transferred to the radiometric data through the radiative transfer processes.

To investigate how this information transfer occurs, let us consider a simple case: a diagonal covariance matrix $\mathbf{W}$ of measurement errors with constant diagonal elements and no a priori information about the parameters ( $\mathbf{C}$ is not considered). In this case, the posterior covariance matrix reduces to:

$$
\Delta=(\text { measurements errors }) \times\left(\mathbf{U}_{\infty}^{\mathrm{T}} \mathbf{U}_{\infty}\right)^{-1}
$$

where $\mathbf{U}^{\mathrm{T}} \mathbf{U}$ is the Fisher information matrix. The amplification factors of the measurement errors for each retrieved parameter are:

$$
\sqrt{\left(\mathbf{U}_{\infty}^{\mathrm{T}} \mathbf{U}_{\infty}\right)^{-1} \delta_{\mathrm{ii}}}
$$

where $\delta_{\mathrm{ii}}$ indicates that we consider only the diagonal elements of the $\left(\mathbf{U}^{\mathrm{T}} \mathbf{U}\right)^{-1}$ matrix, each one corresponding to the parameter $\mathbf{a}_{i}$.

The diagonal terms of $\mathbf{U}^{\mathrm{T}} \mathbf{U}$ represent the sums of the squared sensitivities, while the off-diagonal terms are the sums of the two by two products of the sensitivities representing the correlation between different sensitivities. We examine the Fisher matrix of information instead of the covariance matrix $\Delta$. It is also easier to interpret the correlation coefficient of the sensitivities than to interpret the covariance terms of $\Delta[1]$ :

$$
\rho_{\mathrm{ik}}=\left\{\mathbf{U}^{\mathrm{T}} \mathbf{U}\right\}_{\mathrm{ik}} /\left(\left\{\mathbf{U}^{\mathrm{T}} \mathbf{U}\right\}_{\mathrm{ii}}\left\{\mathbf{U}^{\mathrm{T}} \mathbf{U}\right\}_{\mathrm{kk}}\right)^{1 / 2} .
$$

A correlation coefficient close to zero indicates the information about two parameters to be linearly independent, while a large correlation coefficient $\left(\left|\rho_{\mathrm{ik}}\right| \rightarrow 1\right)$ indicates that the data set cannot resolve separately the parameters $\mathbf{a}_{\mathrm{i}}$ and $\mathbf{a}_{\mathrm{j}}$.

We investigate the information contents of the radiometric measurement of the target vegetation properties by applying the theoretical framework described above. These properties are represented by 7 parameters of a simple model of BRF of the vegetation canopy, which is already described elsewhere [3]. Three of them are optical parameters: the ground albedo $R_{S}$, the bi-Lambertian leaf reflectance and transmittance $r_{L}$ and $t_{L}$. The four remaining parameters are architectural ones: the LAI (Leaf Area Index) L, statistically estimated radius of the circular sun fleck $\mathrm{r}_{\mathrm{SF}}$, two parameters of Kuusk's elliptical leaf distribution function $\theta_{\mathrm{m}}$ and $\varepsilon$ [6]. For $\varepsilon=0$, the leaf angle distribution is isotropic, and for $\varepsilon=1$, all the leaves have the exact inclination $\theta_{\mathrm{m}}$.

The radiative transfer balance is expressed as:

$$
\begin{aligned}
\mu \frac{\partial \mathrm{I}(\mathrm{L}, \underline{\Omega})}{\partial \mathrm{L}}= & -\hat{\mathrm{G}}(\underline{\Omega}) \mathrm{u}_{\mathrm{L}} \mathrm{I}(\mathrm{L}, \underline{\Omega}) . \\
& +\iint_{4 \pi} \mathrm{u}_{\mathrm{L}} \frac{\Gamma\left(\underline{\Omega}^{\prime} \rightarrow \underline{\Omega}\right)}{\pi} \mathrm{I}(\mathrm{L}, \underline{\Omega}) \mathrm{d} \underline{\Omega}^{\prime}
\end{aligned}
$$

where $u_{L}$ is the leaf area density, and $\Gamma$ the scattering phase function of the bi-Lambertian leaves [9]. This formulation is valid for visible wavelengths, and can also be applied to the near infrared (NIR). The modified total interaction cross section takes account of both the cross section of a turbid medium $\mathrm{G}(\underline{\Omega})$ as defined by Ross [9] and the hot spot effect [11]. Knyazikhin et al. [5] show that modifying the interaction cross section to account for the hot spot as proposed in equation (8) leads to a violation of the conservation law. We use an approximation of the solution of equation (8), which allows to simulate the measured BRF, although violating the conservation law. Our estimations are based on the derivative of the BRF with respect to the vegetation parameters, computed with $[\mathrm{BRF}(\mathrm{x}+\Delta \mathrm{x})-\mathrm{BRF}(\mathrm{x})] / \Delta \mathrm{x}$. The energy gained by the violation of the conservation law remains the same for $\operatorname{BRF}(x+\Delta x)$ and $\operatorname{BRF}(x)$. Consequently, 
Table I. Numerical values of the parameters for the numerical experiments.

\begin{tabular}{|c|c|c|}
\hline \multirow[b]{2}{*}{ Parameters } & \multicolumn{2}{|c|}{ Parameters values } \\
\hline & Red & NIR \\
\hline Soil albedo $\left(\mathrm{R}_{\mathrm{S}}\right)$ & 0.03 & 0.15 \\
\hline Leaves reflectance $\left(r_{\mathrm{I}}\right)$ & 0.075 & 0.5 \\
\hline Leaves transmittance $\left(\mathrm{t}_{\mathrm{L}}\right)$ & 0.05 & 0.45 \\
\hline hot spot parameter $\left(\mathrm{r}_{\mathrm{F}}\right)^{\mathrm{L}}$ & \multicolumn{2}{|c|}{0.1} \\
\hline $\begin{array}{l}\text { Leaf Area Index (L) } \\
\text { Leaves modal }\end{array}$ & \multicolumn{2}{|c|}{$0.5 ; 1 ; 2 ; 3 ; 10$} \\
\hline inclination $\left(\theta_{\mathrm{m}}\right)$ & \multicolumn{2}{|c|}{$\begin{array}{c}10^{\circ} \text { (planophile); } 45^{\circ} \\
\text { (plagiophile); } 80^{\circ} \text { (extremophile) }\end{array}$} \\
\hline $\begin{array}{l}\text { Leaves distribution } \\
\text { eccentricity }(\varepsilon)\end{array}$ & \multicolumn{2}{|c|}{ 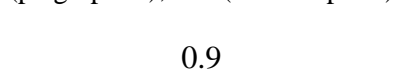 } \\
\hline
\end{tabular}

the exceeding energy is cancelled after the differentiation. The energy gained is about 3\% (red, LAI $=2$ ), irrespective of the sighting direction, except within a narrow solid angle around the hot spot direction. Since the sampled direction does not account for the hot spot direction, it should have no consequence on the correlations terms.

The simulated BRF are expressed as the sum of three different terms (only attenuated radiations, single scattering and multiple scattering). The mathematical expression of the single scattering may be considered separately from the other terms [6], because it contains the most information about the vegetation properties, since it undergoes only one interaction:

$$
\operatorname{BRF}^{1}\left(\underline{\Omega}_{0} \rightarrow \underline{\Omega}\right)=\frac{\Gamma\left(\underline{\Omega}_{0} \rightarrow \underline{\Omega}\right)}{\left|\mu_{0}\right| \mu} \int_{0}^{\mathrm{L}} \mathrm{T}_{0}\left(\mathrm{~L}^{\prime}\right) \mathrm{T}\left(\mathrm{L}^{\prime}\right) \mathrm{dL} \mathrm{L}^{\prime}
$$

where $\mathrm{T}_{0}\left(\mathrm{~L}^{\prime}\right)$ is the transmission of the direct solar radiation through the canopy layers above the level $\mathrm{L}$, and $\mathrm{T}(\mathrm{L}$ ') the transmission of the scattered radiation, and L' the LAI $\left(0<\mathrm{L}^{\prime}<\mathrm{L}\right)$. In the hot spot direction, the optical path is zero, $\mathrm{T}(\mathrm{L})=1$.

The bi-directional reflectance factors (BRF) are deduced from the intensities at the top of the canopy from the relationship $\mathrm{R}(\underline{\Omega})=\pi \mathrm{I}(\underline{\Omega}) / \mu_{0}$, where $\mu_{0}$ is the cosine of the sun zenith angle.

In the next section, we assume that the retrieval has been performed and the retrieved values of parameters are close to those described in Table I.

\section{Results}

\subsection{Conditions of the simulations}

In this paper, we consider only some typical cases, because our goal is to show both the necessity to assess the information contained within the measurements and the role played by the sun-viewing angles configuration. To prepare and analyse an exhaustive data set would be necessary to tackle the optimisation of data sampling under real conditions. The values for the vegetation parameters we used in this study are listed in Table I. For both red
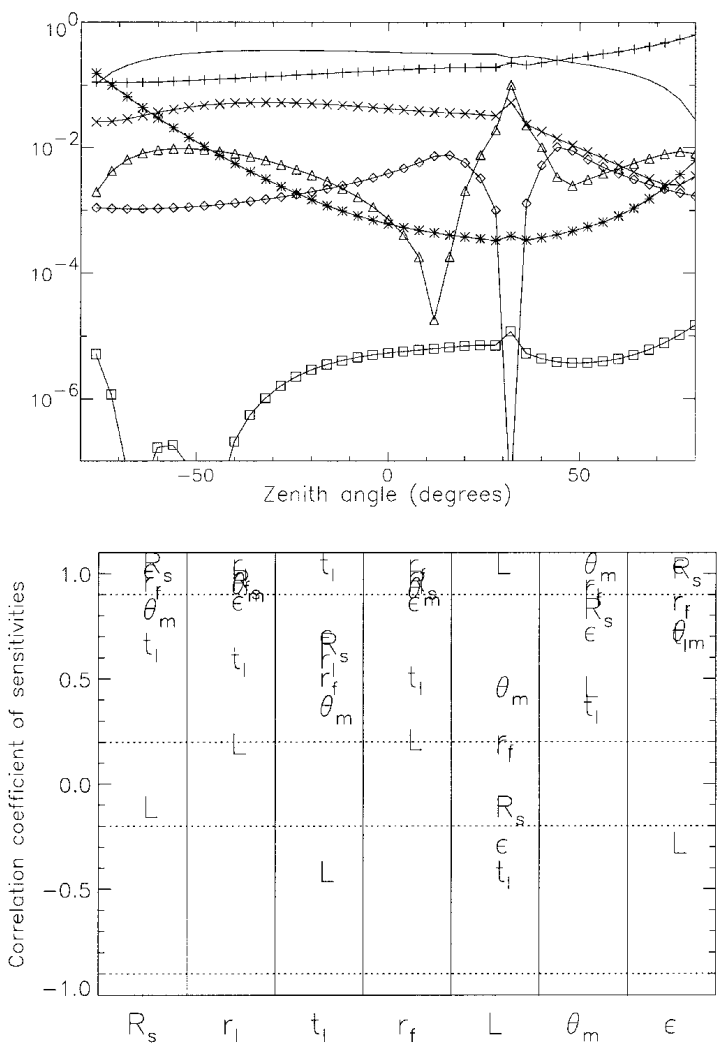

Fig. 1. Squared sensitivities (top) and corresponding crosscorrelations (bottom). LAI $=0.5, \theta_{\mathrm{m}}=10^{\circ}$, red wavelength, sun zenith angle at $32^{\circ}$. The squared sensitivities are computed with respect to the soil albedo $\mathrm{R}_{\mathrm{S}}(-)$, leaves reflectances $r_{\mathrm{L}}(+)$, leaves transmittances $(*)$, hot spot parameter $(\diamond)$, Leaf area index $\mathrm{L}(\Delta)$, leaves modal inclination $\theta_{\mathrm{m}}(\times)$ and the leaves inclination dispersion $\varepsilon(\square)$. 

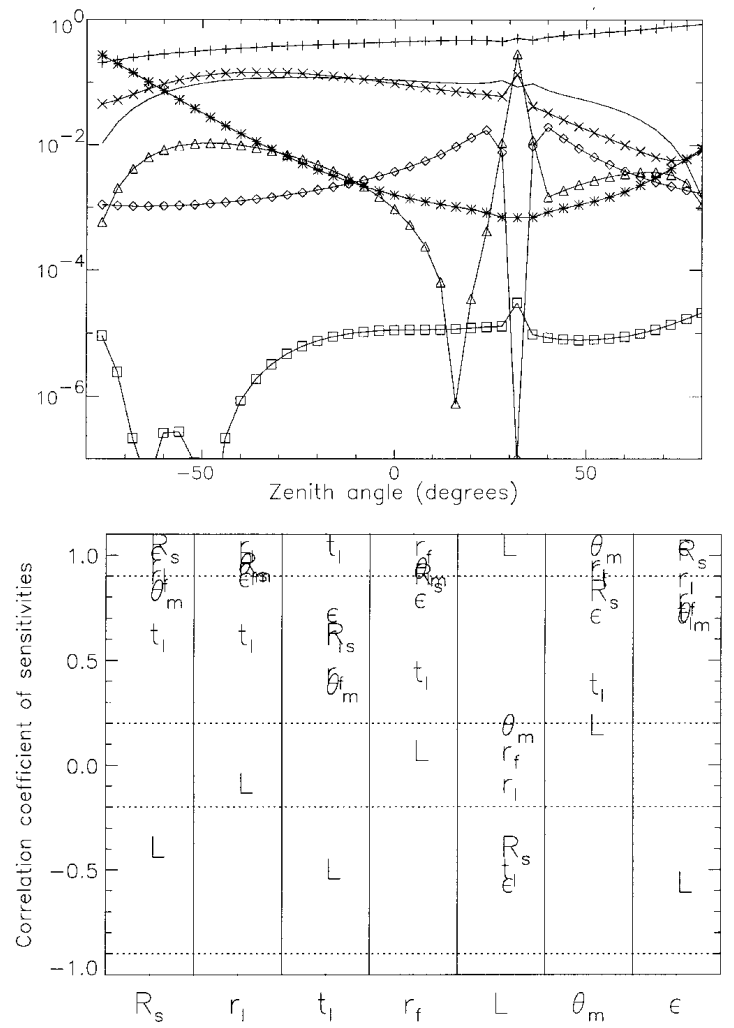

Fig. 2. Squared sensitivities and corresponding cross correlations. $\mathrm{LAI}=1, \theta_{\mathrm{m}}=10^{\circ}$, red wavelength and sun zenith angle at $32^{\circ}$. Same legend as in Figure 1.

wavelengths and near infrared, the soil is chosen darker than the leaves, since we are interested mainly in the retrieval of vegetation properties.

The sun is placed at a zenith angle of $32^{\circ}$. We assume that the synthetic BRF are 'measured' for a single wavelength and for viewing angles in the solar plane from $-76^{\circ}$ to $80^{\circ}$ with a step of $4^{\circ}$. These directions are considered in two parts: a forward scattering direction (azimuth $\varphi=180^{\circ}$ ) noted with negative scattering zenith angle, and backward scattering direction (azimuth $\varphi=0^{\circ}$ ) noted with positive zenith scattering angle. The squared sensitivities of the BRF to the model parameters are computed in the solar plane. The correlation between the sensitivities are computed for a set of measurements sampled at 9 zenith angles (nadir, $\pm 24^{\circ}, \pm 44^{\circ}, \pm 60^{\circ}, \pm 72^{\circ}$ ) in the solar plane.
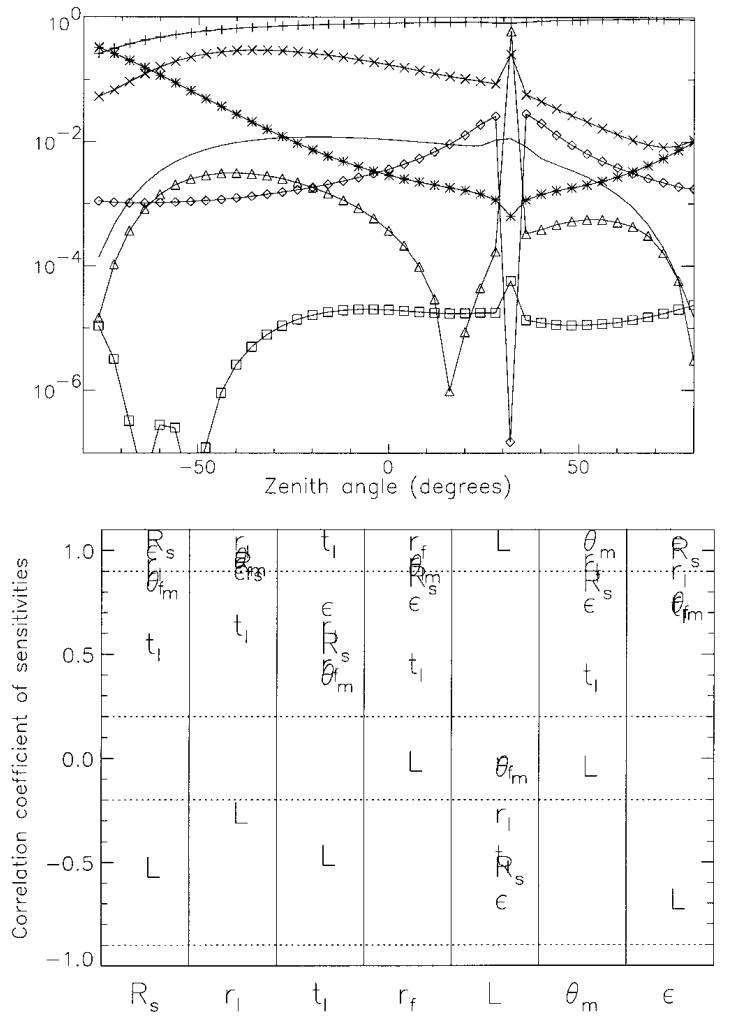

Fig. 3. Squared sensitivities and corresponding cross correlations. $\mathrm{LAI}=2, \theta_{\mathrm{m}}=10^{\circ}$, red wavelength and sun zenith angle at $32^{\circ}$. Same legend as in Figure 1.

\subsection{Physical interpretation}

\subsubsection{Sensitivities}

The squared sensitivities are represented in Figures 1 to 9 , with respect to the observation zenith angle. Figures 1 to 5 represent the squared sensitivities and the corresponding cross correlations (corresponding to the chosen set of 9 angles, c.f. Sect. 3.1) for an increasing LAI, leaves at $10^{\circ}$, a red wavelength and a sun zenith angle of $32^{\circ}$. Figures 6 to 9 represent the same quantities for various conditions.

It should be reminded that we are dealing with the sensitivities of the BRF with respect to the parameters and not with the variations of the parameters themselves. The concept of sensitivity means that the physical processes depending on a 

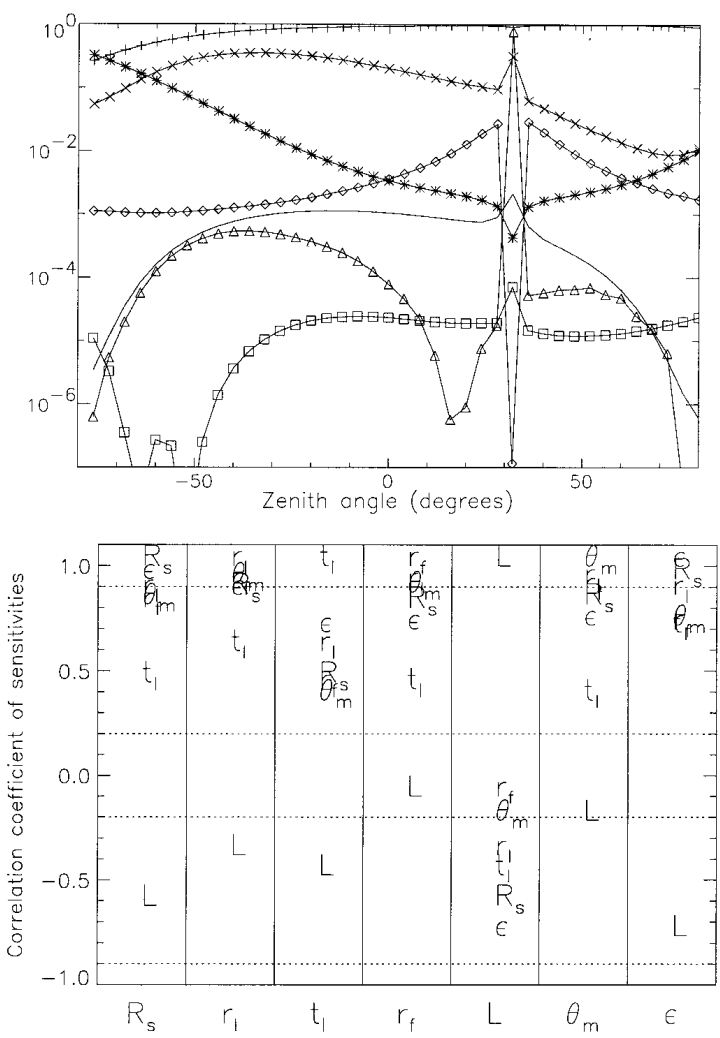

Fig. 4. Squared sensitivities and corresponding cross correlations. $\mathrm{LAI}=3, \theta_{\mathrm{m}}=10^{\circ}$, red wavelength and sun zenith angle $32^{\circ}$. Same legend as in Figure 1.

parameter are observable within the measurements. Let us try to investigate the physical meaning of the information contents by considering the sun beam scattered back by the vegetation layer and the underlying ground. To do so, we consider the behaviour of the information contents as a function of the scattering direction in the solar plane and also as a function of the LAI.

A sun beam penetrating in the vegetation is obviously reflected first by the upper face of the leaves with an albedo $r_{L}$ or transmitted with the coefficient $t_{L}$. Since $r_{L}>t_{L}$, the intensities scattered within the medium depend mainly on the reflectance factor $r_{L}$, especially in the backward directions $\left(\varphi=0^{\circ}\right)$. From backward to forward directions $\left(\varphi=180^{\circ}\right)$, the sensitivity of the BRFs
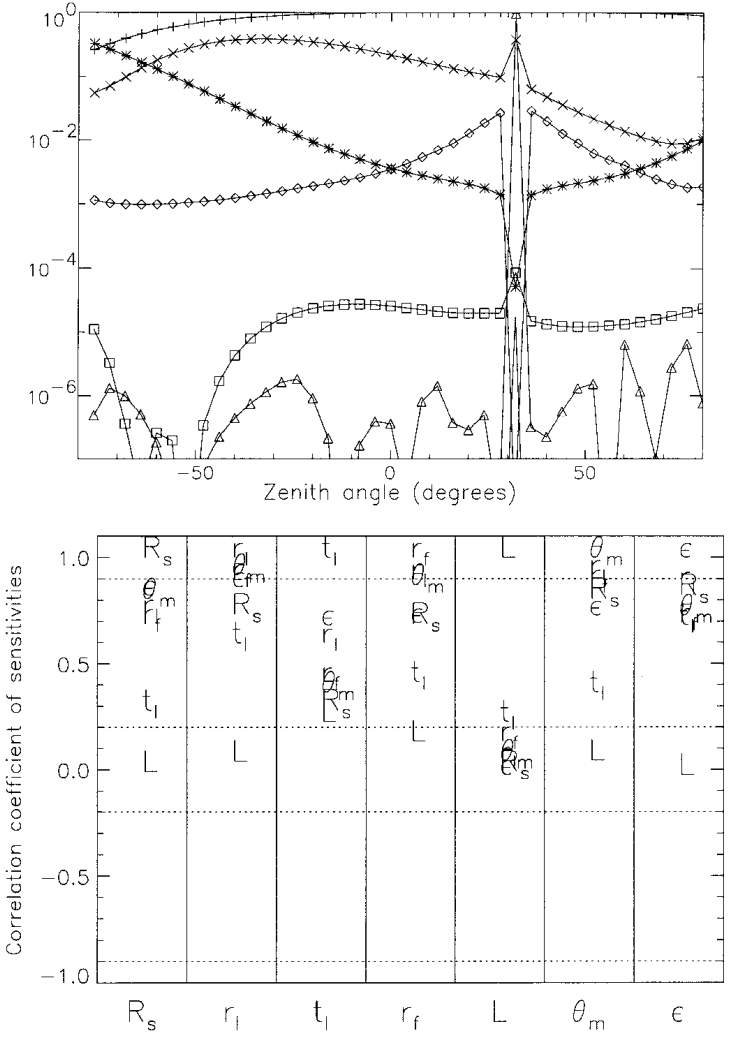

Fig. 5. Squared sensitivities and corresponding cross correlations. LAI $=10, \theta_{\mathrm{m}}=10^{\circ}$, red wavelength and sun zenith angle at $32^{\circ}$. Same legend as in Figure 1.

to $r_{L}$ decreases slightly while the sensitivity to $t_{L}$ increases dramatically when the azimuth scattering angle tends towards $90^{\circ}$ (horizon). Both the sensitivities to $t_{L}$ and $r_{L}$ increase when $L$ increases (i.e. the amount of interaction of the radiation with vegetation increases with the vegetation depth), while the shape of the corresponding curves remain the same with respect to the scattering zenith angle.

The sensitivity to $r_{L}$ increases with LAI. This behaviour makes sense if we consider the soil albedo $\mathrm{R}_{\mathrm{S}}$, and the layer thickness $\mathrm{L}$. The radiation penetrating down is scattered along its path until it reaches the Lambertian ground, where it is scattered with an albedo $R_{S}$. If the layer is thin enough $\left(\mathrm{L}=0.5 \mathrm{~m}^{2} / \mathrm{m}^{2}\right)$, the measured BRFs are mainly sensitive to $R_{S}$, while its sensitivity to the soil albedo decreases as LAI increases. Indeed, the sensitiv- 

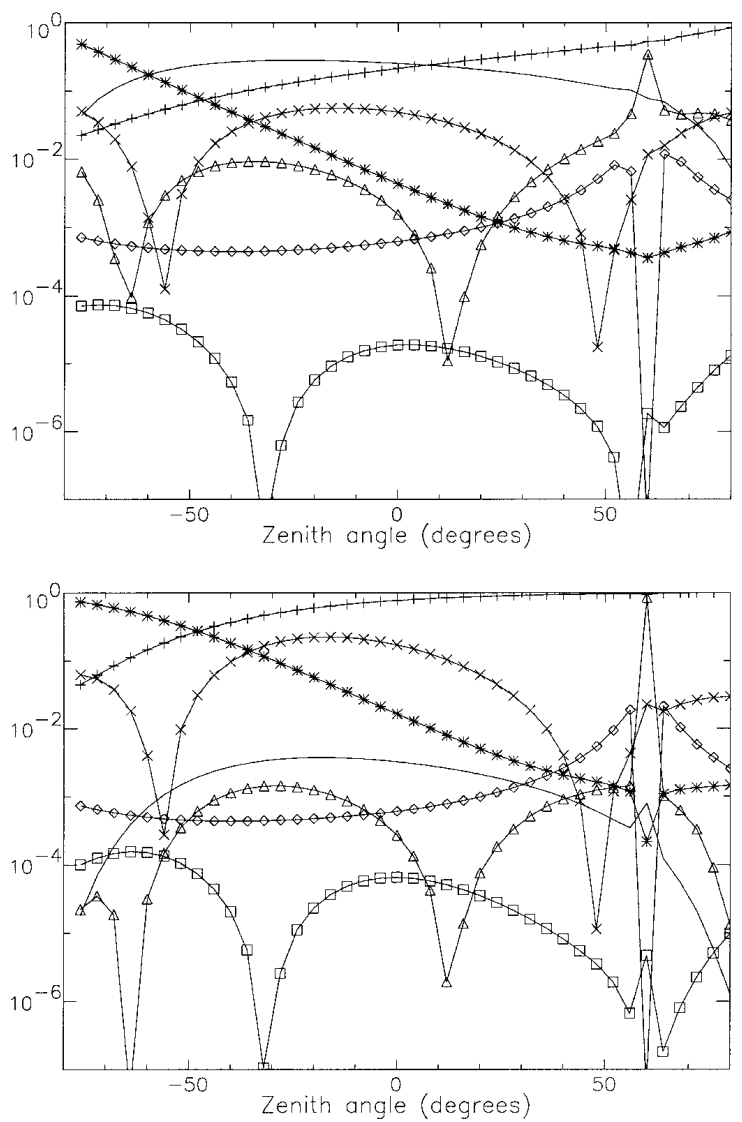

Fig. 6. Squared sensitivities $\mathrm{LAI}=0.5$ and $\mathrm{LAI}=2, \theta_{\mathrm{m}}=10^{\circ}$, red wavelengths, sun at $60^{\circ}$. Same legend as in Figure 1 .

ity to $\mathrm{R}_{\mathrm{S}}$ can be considered as null (lower than 1.E7) for $\mathrm{L}=10 \mathrm{~m}^{2} / \mathrm{m}^{2}$.

The behaviour of these sensitivities should also be considered as a function of the architectural parameters such as the hot spot and the leaf orientations. We first investigated the sensitivities to these architectural parameters, and second we observed the consequences on the optical parameters.

The sensitivity of the BRF to the hot spot parameter $\mathrm{r}_{\mathrm{F}}$ increases ${ }^{\circ}$ ) by a factor of 10 (from 1.E-3 to 1.E-2, approx.) as the zenith viewing angle varies from the horizontal directions $\left(\theta= \pm 90^{\circ}\right)$ to the sun direction $\left(\theta=32^{\circ}, \varphi=0\right.$, but decreases
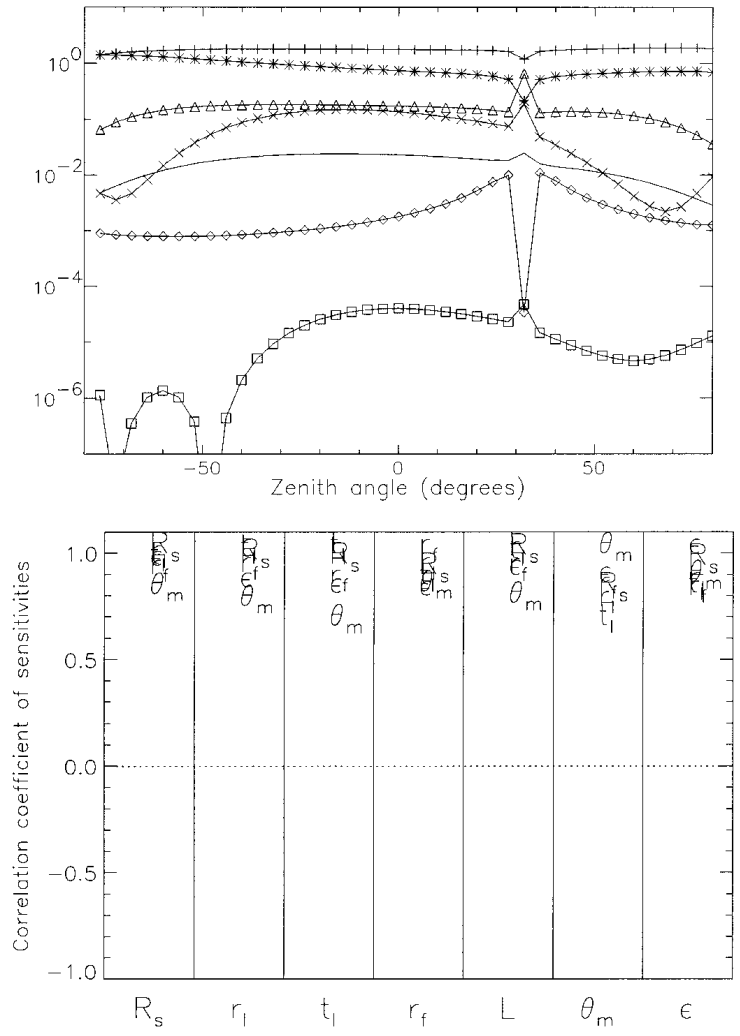

Fig. 7. Squared sensitivities and corresponding cross correlations. $\mathrm{LAI}=2, \theta_{\mathrm{m}}=10^{\circ}$, near infrared wavelength. Same legend as in Figure 1.

suddenly to 0 at the hot spot direction. This behaviour can be explained in the following way. The radiation scattered around the exact sun direction is provided with the information about the hole radius $\left(\mathrm{r}_{\mathrm{F}}\right)$ and this information increases as the zenith angle approaches to the hot spot direction. On the contrary, the radiation that is scattered back into the sun direction does not interact with adjacent scatterrers (leaves in the present case). Consequently, it provides no information about the hole size, which results in null sensitivity. When we take into account the multiple scattering, there is some leaking of the information about the hole size from adjacent directions to the exact sun direc- 

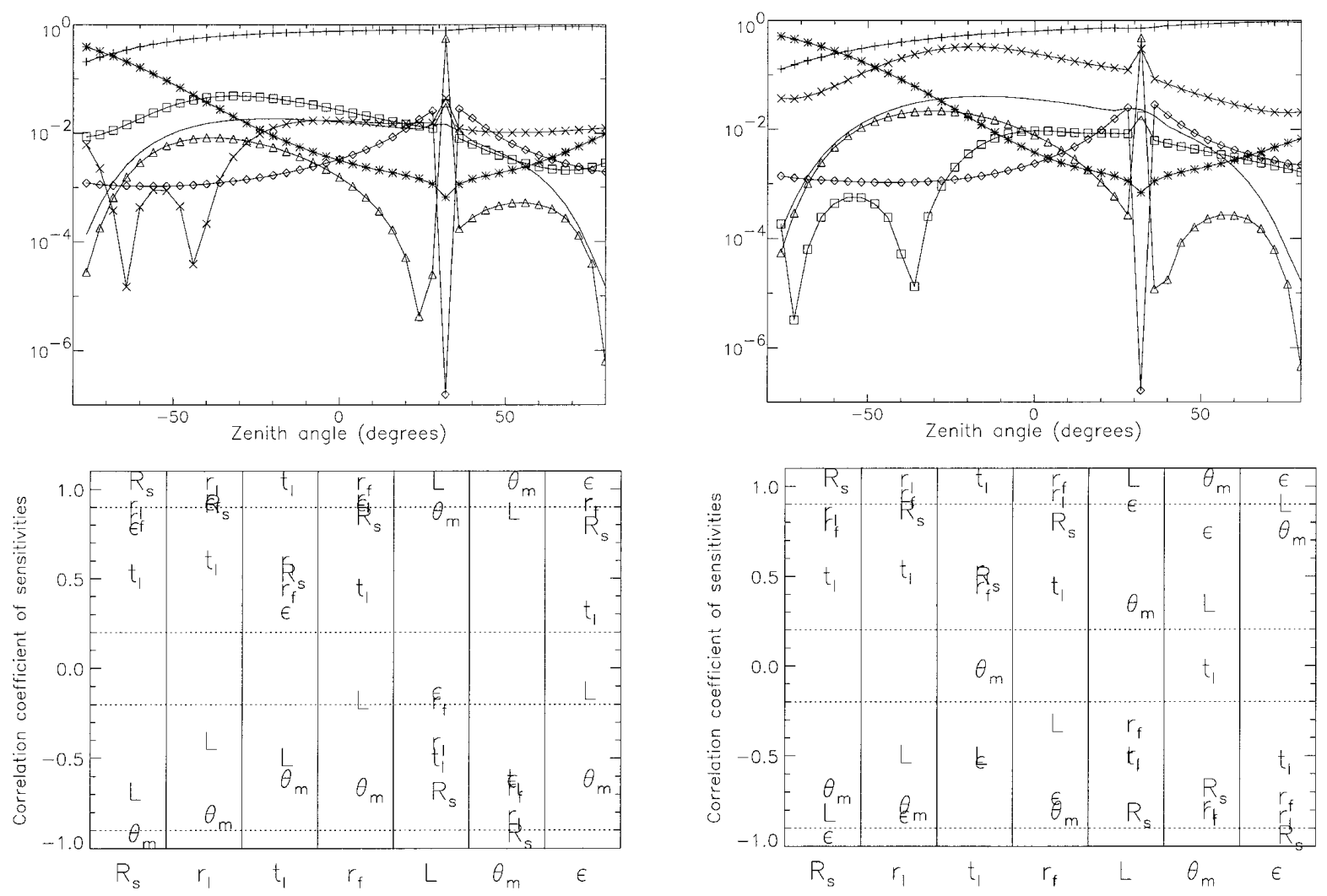

Fig. 8. Squared sensitivities and corresponding cross correlations. $\mathrm{LAI}=2, \theta_{\mathrm{m}}=45^{\circ}$, red wavelength, sun zenith angle at $32^{\circ}$. Same legend as in Figure 1.

tion (about 1.E-7). The decrease of the sensitivity at the exact sun direction is sharper as the LAI increases. This means that the loss of sensitivity for a deep canopy can be considered as a singularity, while this decreasing can be observed on several points for a thin canopy. This specific behaviour of the hot spot may affect the sensitivities of other parameters: the sensitivity to all the other parameters (except the transmittance) exhibits a local maximum in the sun direction, because the medium is not really turbid in this direction.

The sensitivity of the measurements to the LAI increases dramatically in the sun direction, since the BRF depends directly on this parameter in this direction. According to equation (9), the single

Fig. 9. Squared sensitivities and corresponding cross correlations. $\mathrm{LAI}=2, \theta_{\mathrm{m}}=80^{\circ}$, red wavelength, sun zenith angle at $32^{\circ}$. Same legend as in Figure 1.

scattering BRF in the sun direction depends directly on the LAI, since the integral becomes a constant times the LAI.

The sensitivity to the soil albedo $\mathrm{R}_{\mathrm{S}}$ exhibits two local maxima around the hot spot direction for a small LAI (Figs. 1 and 2), but a unique maximum in the sun direction for a larger LAI (Fig. 3). This occurs because the optical path is minimal in the sun direction (principle of the hot spot) and the backscattered radiation has a maximum of information about the underlying soil. The sensitivity with respect to the soil albedo decreases while the viewing zenith angle increases, because for an increasing viewing angle the optical path is more 
important, and the intensities scattered by the soil are more attenuated.

However, all these specific features of the information contents around the sun direction is due to the mathematical formulation we have adopted to represent the hot spot. Even if these findings seem to make sense from physical point of view, they have to be experimentally confirmed.

The orientation of the leaves may have an important effect on the sensitivities and their correlation since they are parts of the scattering phase function $\Gamma$. This function is characterised with two parameters: the leave principal modal angle $\left(\theta_{\mathrm{m}}\right)$ and a parameter $(\varepsilon)$ indicating the dispersion around this angle. The sensitivity with respect to $\theta_{\mathrm{m}}$ is very low (about $1 . \mathrm{E}-6$ to $1 . \mathrm{E}-5$ ) for planophile $\left(\theta_{\mathrm{m}}=10^{\circ}\right)$ for all directions and all values of the LAI (see Figs. 1 to 5, and Fig. 7). However, this sensitivity is more important for $\theta_{\mathrm{m}}=45^{\circ}$ and $\theta_{\mathrm{m}}=80^{\circ}$. It is also noticeable that this sensitivity for $\theta_{\mathrm{m}}=10^{\circ}$ is more important with a sun zenith angle of $60^{\circ}$ (see Fig. 6), the squared sensitivities being increased by a factor of 10 . The more the principal direction of the leaves is far from the normal direction to the sun light, the more the measured BRFs are sensitive to $\theta_{\mathrm{m}}$. The squared sensitivity with respect to the parameter $\varepsilon$ are important in all the cases. Both of these sensitivities exhibit a little peak in the sun direction, because in this direction $T\left(L^{\prime}\right)=1$ (Eq. (9)), the influence of the scattering phase function (and the leaf orientation parameters) is maximal.

\subsubsection{Correlation between the sensitivities}

In the following, $\mathrm{S}(\mathrm{x})$ designates the sensitivity of the BRFs to the parameter $x$, and $\rho(x, y)$ the correlation of $S(x)$ with $S(y)$. The correlation between the sensitivities are plotted in Figures 1 to 9 simultaneously with the corresponding sensitivity curves described in the previous section. These figures represent the correlation $\rho(x, y)$ where each column corresponds to the parameter $\mathrm{x}$, and the plotted symbols $y$ in each column represent the correlation $\rho(x, y)$. The variables $x$ and $y$ correspond to the model parameters (names referenced in Tab. I).
In Figures 1 to 4 , the correlation coefficient $\rho\left(R_{S}, L\right)$ is always negative, which means that the vegetation prevents the observation of the underlying soil. The more the radiation is scattered by the vegetation, the less radiation reaches the soil and brings back information about $\mathrm{R}_{\mathrm{S}}$. The sensitivity to $\mathrm{L}$ increases at the expense of the sensitivity to $R_{S}$. Consequently, the correlation of the sensitivities with respect to $R_{S}$ and to $L$ is negative, is small for $\mathrm{L}=0.5$, moderate for $\mathrm{L}=1,2$ or 3 , and null for $\mathrm{L}=10 \mathrm{~m}^{2} / \mathrm{m}^{2}$. In the case of the near infrared wavelength, the leaves are more transparent (only $5 \%$ attenuation per leaf) than for the red wavelength. Thus, an optically thin canopy (Fig. 7) corresponds to a multiple source of illumination for the underlying ground, resulting in a positive correlation.

The correlation $\rho\left(r_{L}, L\right)$ is positive for a thin layer of vegetation. Evaluating the LAI can be regarded as counting the number of leaves (or the number of interactions with leaves) in the vertical direction. The positive correlation is possible if the single scattering is more important than multiple scattering, because the single scattering provides information about L (see Eq. (9) and previous section). For a thicker layer (i.e. for larger multiple scattering), the sensitivity to $\mathrm{L}$ decreases as the sensitivity to $r_{L}$ increases. In this case, the major part of the measured radiation is scattered back in the upper layers of the vegetation, and little radiation can come back from the deeper vegetation layer which provides information about the whole vegetation layer, i.e. the LAI.

In most of the cases, the correlation coefficient $\rho\left(t_{L}, L\right)$ is negative because the radiation transmitted by leaves can be observed only if it is scattered back. This process requires at least 2 interactions between a photon and the leaves (or one with a leave and one with the ground). The multiple scattering results in general in filtering the information about all the parameters. Consequently it does not provide any information (in term of sensitivity) about a given parameter. A positive value of $\rho\left(t_{L}\right.$, L) exceptionally occurs for a very large LAI. Little information about $\mathrm{L}$ contained within the multiple scattering should be used to limit a possible inter- 
val to L. Consequently, this information cannot be separated from the sensitivity to $t_{L}$.

The leaves principal angle $\left(\theta_{\mathrm{m}}\right)$ and dispersion parameter $(\varepsilon)$ should be considered together. In the case of a thin vegetation layer, the radiation is scattered by oriented leaves along the whole optical path and the single scattering radiations (Eq. (9)) are not too much attenuated. For a larger LAI, the number of interaction with the vegetation is more important (multiple scattering increases), and the effect of the orientation of the leaves may be smoothed out. In a particular case with $\mathrm{r}_{\mathrm{L}} \approx \mathrm{t}_{\mathrm{L}}$, a biLambertian leaf exhibits rather isotropic scattering characteristics. Then, the information about the LAI takes much importance than information about the leaves orientation.

For the near infrared radiations, the BRF seems to be more sensitive to the leaf and soil parameters (Fig. 7), because of the larger values of optical properties of the leaves and soil (Tab. I). Moreover, the sensitivities exhibit less dependence on the scattering direction. As a consequence, the corresponding correlations are all particularly important and positive, because the relationship between one sensitivity curve to the other is almost linear.

\subsection{Errors of the parameters to retrieve}

The amplification factors (Eq. (6)) of the measurement errors have been evaluated for each parameter to retrieve, for $\mathrm{LAI}=0.5, \mathrm{LAI}=2.0$ and LAI $=10$ (Fig. 10), each time for a red wavelength, a sun zenith angle of $32^{\circ}$, and leaves oriented at $\theta_{\mathrm{m}}=10^{\circ}$.

The error of the soil albedo parameter $\left(R_{S}\right)$ increases with the leaf area index, because the vegetation layer attenuates the radiations scattered by the ground. For the LAI of $10 \mathrm{~m}^{2} \cdot \mathrm{m}^{-2}$, the amplification factor of $R_{S}$ has not been reported (its value is about $4.2 \times 10^{5}$ ) in order to keep the y-range of the plots readable. It seems that the soil properties may not be accurately retrieved for LAI equal to $2 \mathrm{~m}^{2} \cdot \mathrm{m}^{-2}$ or greater, for a visible wavelength.

The amplification factor of the leaf reflectance $\mathrm{r}_{\mathrm{L}}$ increases from LAI $=0.5$ to $\mathrm{LAI}=2$ and

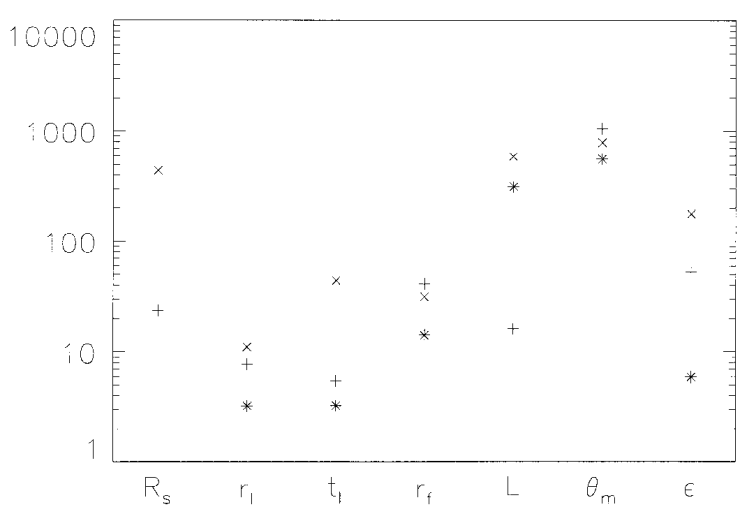

Fig. 10. Amplification factor of the measurements errors, with respect to the model parameters. LAI $=0.5(+), \mathrm{LAI}=2(\times)$, and LAI $=10\left(_{*}\right), \theta_{\mathrm{m}}=10^{\circ}$, red wavelength, sun zenith angle at $32^{\circ}$.

decreases from LAI $=2$ to LAI $=10$. This corresponds to the sign of the correlation factor: positive for LAI $=0.5$ and LAI $=10$ (Figs. 1 and 5 , respectively), but negative for $\mathrm{LAI}=1$ to $3 \mathrm{~m}^{2} \cdot \mathrm{m}^{-2}$ (Figs. 2, 3, and 4). Therefore, the leaf reflectance $\left(r_{L}\right)$ may be retrieved for any value of LAI. The same behaviour can be observed for the leaf transmittance $\left(t_{L}\right)$. However, the amplification factor for LAI $=2$ is quite important (about 45 times the measurements errors) and may make the retrieval of $t_{L}$ impossible if the measurements errors are too important.

The errors related to the hot spot parameter $\left(\mathrm{r}_{\mathrm{SF}}\right)$ increase with the layer thickness, and remain important (greater than 10 times the measurements errors) for all the cases.

The amplification of errors related to the LAI is important for LAI $=2$ or greater, because the layer thickness becomes optically too thick. The fact that the amplification factor for LAI $=10$ (errors multiplied by about 300) is lower than for LAI $=2$ (amplification factor about 600) comes from the low pair correlation between the sensitivities to the LAI and to the other parameters (Fig. 5).

The amplification factors related to the leaf orientation $\theta_{\mathrm{m}}$ is particularly important, since the 
sensitivity with respect to $\theta_{\mathrm{m}}$ is low at $\theta_{\mathrm{m}}=10^{\circ}$ (see Figs. 1 to 5). Concerning the error related to the parameter $\varepsilon$, the accuracy is better for a very thick canopy $(\mathrm{LAI}=10)$.

We estimated the amplification factors of the measurement errors by assuming that the solution of the inverse problem has been reached and is close to the values provided in Table I. The result of the above analysis provide a physically sound picture of the retrievability of the vegetation parameters. The next step should be to perform the retrieval from real data before determining the measurement errors amplification factors of the retrieved parameters, since the retrieval of some parameters (especially those corresponding to the leaf orientation) might not be achieved. However, the presented method would be still valid.

\section{Conclusion}

In this paper, a general mathematical framework, based on the Fisher information matrix, has been introduced. This framework enables the assessment of the information contents related to the target parameters provided by the measurements. The information contents and the accuracy of the retrieved parameters are closely related one to the other. Clearly, the more a measurement contains information the more the parameters are accurately retrieved. The formalism shows that the information is based both on the sensitivity of the measurements with respect to the parameters to retrieve and the pair correlation of theses sensitivities. A simple model of the bi-directional reflectance factors (BRFs) scattered by the vegetation has been used to investigate the sensitivities of the BRFs with respect to the vegetation parameters and the corresponding pair correlations. The physical interpretation of these quantities provides an interesting insight into the interaction of the radiations with the vegetation properties. We show that the sensitivity of the BRFs with respect to the parameters and their correlation vary with the looking direction and depend on the vegetation properties and the wavelength. The present analysis shows clearly that we should consider not only a high sensitivity with respect to the desired parameters, but also the correlation between the measurements.

Furthermore, the variances of the retrieved parameters (i.e. the 'error bars') have been studied in terms of amplification factors of the measurement errors. These terms are directly related to the Fisher information matrix. It appears clearly that the accuracy of the retrieved parameters depends both on the sensitivity of the measured BRF to the vegetation parameters and correlations between these sensitivities. Thus the precision of the retrieved parameters is limited both by the feasibility (studied in this paper) of the retrieval and by the measurements sampling. More precisely, we show that the looking angles have to be chosen in such a way that the BRFs are sensitive enough to the parameter but not too much correlated between them.

The model considered in this paper simplifies soil and leaf reflection: directional properties of soil reflectance and specular reflection of leaves are omitted. Considering more realistic soil reflectance or leaf reflection means that we should consider more parameters to describe the soil. As shown in the paper, parameters may be quite correlated to each other, and the resulting accuracy of the sought parameters should decrease if too many parameters are included within the model.

Thus we have to deal with this compromise: choosing a model with the most reduced set of parameters, and including parameters to simulate the data with the best precision. We look for the best model in principle, but the notion of best model depend on the considered task. For example, our model should be modified if we apply it to a very sparse vegetation where the soil contribution to the BRF is very large. When the 'best model' is chosen, the matrix $\mathbf{W}$ should account for the measurement error and the model errors. Then, if the modified model does not account for parameters describing the soil reflectance, the corresponding uncertainty should be evaluated.

The present study was conducted with a limited scope to show what an analysis of information contents can provide about the feasibility and accuracy 
of retrieval of vegetation parameters. Nevertheless, this formalism may be used to perform an optimisation of radiometers (number and directions of the viewing angles and/or wavelengths), but also to investigate the adequacy between an inverse model involved in the retrieval procedure and available observation data.

Acknowledgements: This study was conducted jointly under Japanese Space Agency (NASDA) grant, GLI/ADEOS II project No. A2GRF005, and French National Institute for Sciences of the Universe (INSU) grant, 96/ATP/494/220.2.

\section{References}

[1] Dubovik O.V., Lapyonok T.V., Oshchepkov S.L., Improved technique for data inversion: optical sizing of multicomponent aerosols, Appl. Optics 34 (1995) $8422-8436$.

[2] Gobron N., Pinty B., Verstraete M.M., Theoretical limits to the estimation of the leaf area index on the basis of visible and near-infrared remote sensing data, IEEE Trans. Geosci. Remote Sens. 35 (1997) 1438-1445.

[3] Iaquinta J., Pinty B., Adaptation of a bidirectional reflectance model including the hot-spot to an optically thin canopy, Proc. Sixth Int. Symp. "Physical measurements and signatures in remote sensing", Val d'Isère, France, 1994, pp. 683-690.

[4] Knyazikhin Y., Martonchik J.V., Diner D.J., Myneni R.B., Verstraete M., Pinty B., Gobron N.,
Estimation of vegetation canopy leaf area index and fraction of absorbed photosynthetically active radiation from atmosphere-corrected MISR data, J. Geophys. Res. 103 (1998), pp. 32239-32256.

[5] Knyazikhin Y., Martonchik J.V., Myneni R.B., Diner D.J., Running S.W., Synergistic algorithm for estimating vegetation canopy leaf are index and fraction of absorbed photosynthetically active radiation from MODIS and MISR data, J. Geophys. Res. 103 (1998) 32 257-32276.

[6] Kuusk A., A fast, invertible canopy reflectance model, Remote Sens. Environ. 51 (1995) 342-350.

[7] Privette J.L, Myneni R.B., Tucker C.J., Emery W.J., Invertibility of a 1D discrete ordinates canopy reflectance model, Remote Sens. Environ. 48 (1994) 89-105.

[8] Privette J.L., Myneni R.B., Emery W.J., Hall F.G., Optimal sampling conditions for estimating grassland parameters via reflectance model inversions, IEEE Trans. Geosci. Remote Sens. 34 (1996) 272-284.

[9] Ross J., The Radiation Regime and Architecture of Plant Stands, Junk, The Hague, 1981.

[10] Tarantola A., Inverse Problems Theory. Methods for Data Fitting and Model Parameter Estimation, Elsevier, 1987.

[11] Verstraete M.M., Pinty B., Dickinson R., A physical model of the bidirectional reflectance of vegetation canopies. 1. Theory, J. Geophys. Res. 95 (1990) 11755-11765.

[12] Verstraete M.M., Pinty B., Myneni R.B., Potential and limitations of information extraction on the terrestrial biosphere from satellite remote sensing, Remote Sens. Environ. 58 (1996) 201-214. 